\title{
Association between anemia and hematological indices with mortality among cardiac intensive care unit patients
}

\author{
Hamza A. Rayes ${ }^{1}$ - Saraschandra Vallabhajosyula ${ }^{1,2} \cdot$ Gregory W. Barsness ${ }^{2} \cdot$ Nandan S. Anavekar $^{2} \cdot$ Ronald S. Go $^{3}$. \\ Mrinal S. Patnaik ${ }^{3} \cdot$ Kianoush B. Kashani $^{1,4} \cdot$ Jacob C. Jentzer ${ }^{1,2} \mathbb{B}$
}

Received: 2 July 2019 / Accepted: 11 September 2019 / Published online: 18 September 2019

○) Springer-Verlag GmbH Germany, part of Springer Nature 2019

\begin{abstract}
Background Anemia and elevated red cell distribution width (RDW) or mean corpuscular volume (MCV) are associated with an adverse prognosis in patients with cardiovascular disease and critical illness. Limited data exist regarding these associations in unselected cardiac intensive care unit (CICU) patients.

Methods Retrospective cohort study of CICU patients between January 1, 2007, and December 31, 2015, with a hemoglobin $\mathrm{Hb})$ level measured at admission. Multivariable regression was performed to determine predictors of hospital mortality, and Kaplan-Meier analysis was used to determine post-discharge survival.

Results We included 9644 patients with a mean age of $67.5 \pm 15.1$ years, including 3604 (37.4\%) females. The median (IQR) values of $\mathrm{Hb}, \mathrm{MCV}$ and RDW were $12.2 \mathrm{~g} / \mathrm{dL}(10.6,13.7), 90.7 \mathrm{fL}(87.3,94.2) \mathrm{fL}$, and 14.1\% (13.3, 15.8), respectively. Anemia (admission $\mathrm{Hb}<12 \mathrm{~g} / \mathrm{dL}$ ) was present in 4434 (46\%) patients. A total of $845(8.8 \%)$ patients died in the hospital. Patients with anemia had higher hospital mortality (11.3\% vs. 6.6\%, unadjusted OR 1.82, 95\% CI 1.58-2.10, $p<0.001)$. After multivariable regression, admission $\mathrm{Hb}$ and MCV were not significantly associated with hospital mortality (both $p>0.1$ ), while admission RDW (adjusted OR 1.12 per 1\%, 95\% CI 1.07-1.18, $p<0.001$ ) was significantly associated with hospital mortality. Hospital survivors with lower Hb, higher MCV, or higher RDW had lower post-discharge survival.

Conclusion Elevated RDW on admission was independently associated with higher hospital mortality in CICU patients. These data emphasize the importance of hematologic abnormalities for mortality risk stratification in CICU populations.
\end{abstract}

Hamza A. Rayes and Jacob C. Jentzer contributed equally to this work.

Electronic supplementary material The online version of this article (https://doi.org/10.1007/s00392-019-01549-0) contains supplementary material, which is available to authorized users.

Jacob C. Jentzer

jentzer.jacob@mayo.edu

Hamza A. Rayes

hamzarayes@outlook.com

Saraschandra Vallabhajosyula

vallabhajosyula.saraschandra@mayo.edu

Gregory W. Barsness

barsness.gregory@mayo.edu

Nandan S. Anavekar

anavekar.nandan@mayo.edu

Ronald S. Go

go.ronald@mayo.edu

Mrinal S. Patnaik

patnaik.mrinal@mayo.edu
Kianoush B. Kashani

kashani.kianoush@mayo.edu

1 Division of Pulmonary and Critical Care Medicine, Department of Medicine, Mayo Clinic, 200 First Street SW, Rochester, MN 55905, USA

2 Department of Cardiovascular Medicine, Mayo Clinic, 200 First Street SW, Rochester, MN 55905, USA

3 Division of Hematology and Oncology, Department of Medicine, Mayo Clinic, Rochester, MN, USA

4 Division of Nephrology and Hypertension, Department of Medicine, Mayo Clinic, Rochester, MN, USA 


\section{Graphic abstract}

\section{Unselected Cardiac Intensive Care Unit Patients}

\section{Complete blood count}

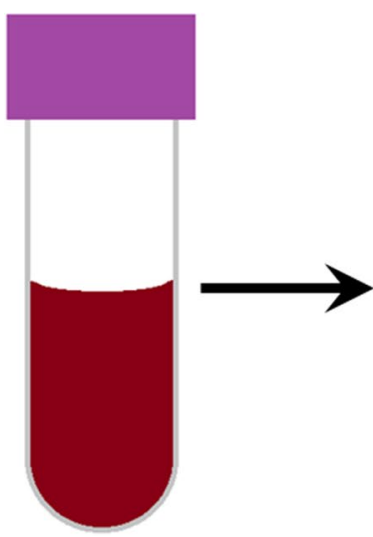

Laboratory findings

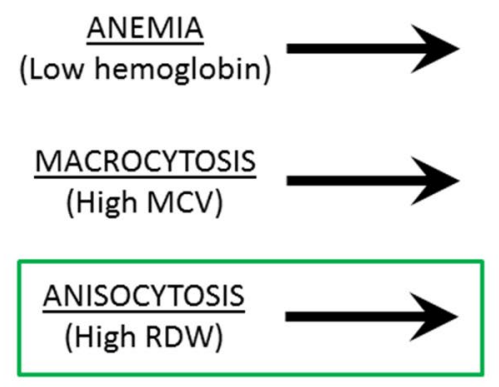

Significant after multivariable adjustment

\section{Higher hospital mortality}

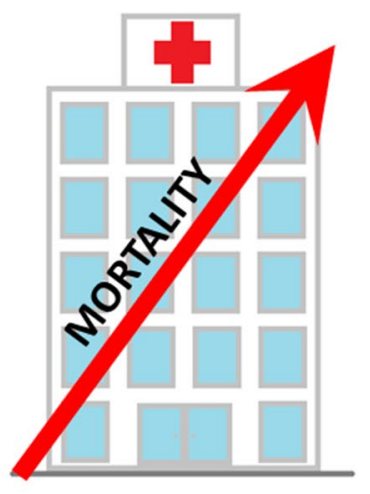

Keywords Red cell distribution width $\cdot$ Anemia $\cdot$ Mean corpuscular volume $\cdot$ Cardiac intensive care unit $\cdot$ Coronary care unit · Mortality

\section{Introduction}

Anemia is common among acutely ill cardiac patients, with a prevalence of up to $19 \%$ and up to $48 \%$ among patients with acute coronary syndrome (ACS) and acute heart failure (HF), respectively $[1,2]$. Anemia is an independent predictor of adverse cardiovascular events, bleeding, and mortality in patients with acute cardiac disease [1-5]. In addition, abnormal values of hematological indices, including red cell distribution width (RDW) and mean corpuscular volume (MCV) have also been associated with worse outcome in patients with acute cardiac disease [6-12]. In general intensive care unit (ICU) populations, the presence of anemia has been associated with demand myocardial ischemia, ICU readmission, and increased mortality [13-15]. As in patients with cardiac disease, elevated RDW and MCV are also associated with worse outcomes in general ICU patients [16-18].

Despite the growing similarities between the cardiac intensive care unit (CICU) and medical ICU populations, CICU patients remain distinct due to their more prevalent cardiac comorbidities [19]. It remains uncertain whether predictors of mortality in general ICU patients without acute cardiac disease or in non-ICU patients with acute cardiac disease would apply similarly to the specialized CICU population. There is a paucity of published data available to describe the association between anemia, RDW, and MCV and adverse outcomes in CICU patients. This study was designed to test the hypothesis that anemia and abnormal values of RDW and MCV are associated with higher mortality in CICU patients.

\section{Methods}

\section{Study population}

This study was approved by the Institutional Review Board of Mayo Clinic as posing minimal risk to patients and was conducted under a waiver of informed consent (IRB \# 16-000722). We retrospectively analyzed a database of consecutive unique adult (aged $\geq 18$ years) patients admitted to the CICU at Mayo Clinic Hospital St. Mary's Campus whose entire CICU admission occurred between January 1, 2007, and December 31, 2015 , as previously reported [20-22]. We only analyzed data from the first CICU admission during the study period to avoid mortality bias associated with CICU readmissions. According to Minnesota state law statute 144.295 , patients must provide authorization to be included in observational research studies; patients who did not have Minnesota Research Authorization were excluded from the database. Patients who did not have available data for admission hemoglobin $(\mathrm{Hb})$ level were excluded from this study.

\section{Data sources}

Demographic, vital sign, laboratory, and other clinical and outcome data were extracted electronically from the medical 
record, including procedures and therapies performed during the CICU and hospital stay. The admission value of all vital signs, clinical measurements, and laboratory values was used, defined as either the first value recorded after CICU admission or the value recorded closest to CICU admission. Although the WHO defines anemia as an admission $\mathrm{Hb}$ level $<12 \mathrm{~g} / \mathrm{dL}$ in women and $<13 \mathrm{~g} / \mathrm{dL}$ in men, we chose an operational definition of anemia as admission $\mathrm{Hb}<12 \mathrm{~g} / \mathrm{dL}$ regardless of gender [23]. Microcytosis was defined as an MCV $<80 \mathrm{fL}$ and macrocytosis was defined as an MCV $>100 \mathrm{fL}$ [23]. The Acute Physiology and Chronic Health Evaluation (APACHE)-III score, APACHE-IV predicted hospital mortality and Day 1 Sequential Organ Failure Assessment (SOFA) score were calculated for all patients using data from the first $24 \mathrm{~h}$ of CICU admission, with missing variables imputed as normal as the default; the mean and maximum values of all daily SOFA scores while the patient remained in the CICU were recorded [20-22, 24, 25]. The Charlson Comorbidity Index (CCI) and individual comorbidities were determined based on a previously-validated electronic algorithm [26]. Discharge diagnoses were identified using International Classification of Diseases (ICD)-9 and ICD-10 diagnosis codes, these diagnoses were not mutually exclusive, and the primary discharge diagnosis could not be determined.

\section{Statistical analysis}

The primary outcome was all-cause hospital mortality, and the secondary outcomes were CICU mortality and 5-year post-discharge mortality, based on the electronic review of the medical record. Groups were compared using Student $t$ test and ANOVA for continuous variables and Pearson Chi-square test for categorical variables. Logistic regression was used to calculate the unadjusted odds ratio (OR) and 95\% confidence interval (CI) values for hospital mortality. Optimal cutoffs for predicting hospital mortality were determined from receiver-operator characteristic curves based on the highest value of Youden's J index (sensitivity + specificity -1 ). Multivariable analysis was performed to determine adjusted OR values for hospital mortality using an adaptive elastic net penalized logistic regression model, with candidate variables including demographics, comorbidities, illness severity, and CICU therapies and complications; an interaction term between $\mathrm{Hb}$ and RDW was included in the model [27]. Optimal tuning parameters for the elastic net were selected by linear grid search in conjunction with tenfold cross-validation to maximize the AUROC. Kaplan-Meier survival analysis was used to compare 5-year post-discharge survival among patients surviving to hospital discharge (hospital survivors), with groups compared using the log-rank test. Cox proportional-hazards analysis was used to determine the associations between $\mathrm{Hb}, \mathrm{MCV}$, and RDW with post-discharge mortality after adjusting for predictors of hospital mortality. Two-tailed $p$ values $<0.05$ were considered statistically significant. Statistical analyses were performed using JMP version 13.0 Pro (SAS Institute, Cary, NC).

\section{Results}

\section{Study population and baseline characteristics}

We screened 12,904 CICU admissions and excluded 2900 as previously described, leaving 10,004 patients in the database [20-22]. An additional 360 patients were further excluded due to lack of available data on admission $\mathrm{Hb}$ level, leaving 9644 patients in the final study population (Supplemental Fig. 1). The median age of the study population was 69.1 years (IQR 57.8, 78.9) and comprised 3604 (37.4\%) females. The median CCI was 2 (IQR 0, 4), with a median APACHE-III score of 58 (IQR 45, 74) corresponding to a median APACHE-IV predicted mortality of 9.5\% (IQR 4.0, 22.8). The median admission $\mathrm{Hb}$ level was $12.2 \mathrm{~g} / \mathrm{dL}$ (IQR 10.6, 13.7); 4434 (46.0\%) patients met our operational definition of anemia (admission $\mathrm{Hb}<12 \mathrm{~g} / \mathrm{dL}$ ), while $5402(56.0 \%)$ met the WHO gender-specific definition of anemia ( $46.3 \%$ of women and $53.7 \%$ of men). Data on MCV were available in 9409 (97.5\%) patients (Supplemental Fig. 1), and the median MCV was $90.7 \mathrm{fL}$ (IQR 87.3, 94.2). Data on RDW were available in 9405 (97.5\%) patients (Supplemental Fig. 1), and the median RDW was 14.1\% (IQR 13.3, 15.6). Patients with anemia were older, more frequently female, had higher rates of comorbidities, greater illness severity and increased utilization of CICU therapies (all $p<0.05$ ) compared to patients without anemia (Table 1); in addition, patients with anemia were more likely to have a discharge diagnosis of HF and less likely to have a discharge diagnosis of ACS. Significant differences were observed in baseline characteristics, comorbidities, illness severity and CICU therapies across RDW quartiles, reflecting higher illness severity, more extensive comorbidities, lower $\mathrm{Hb}$ and greater use of CICU therapies in patients with higher RDW (Supplemental Table 1); the prevalence of HF increased and the prevalence of ischemic heart disease decreased with rising RDW quartile. Patients with a discharge diagnosis of HF had lower Hb (11.7 vs. $12.4 \mathrm{~g} / \mathrm{dL})$ and higher RDW (15.7\% vs. $14.2 \%)$, while patients with a discharge diagnosis of ACS had higher $\mathrm{Hb}$ (12.5 vs. $11.9 \mathrm{~g} / \mathrm{dL})$ and lower RDW (14.1\% vs. $15.3 \%)$ (all $p<0.001$ ); MCV differed minimally between these groups. 
Table 1 Baseline characteristics of the study population, patients with and without anemia (admission hemoglobin $<12 \mathrm{~g}$ / dL)

\begin{tabular}{|c|c|c|c|c|}
\hline & $\begin{array}{l}\text { Overall popula- } \\
\text { tion }(n=9644)\end{array}$ & $\begin{array}{l}\text { Patients without } \\
\text { anemia }(n=5210)\end{array}$ & $\begin{array}{l}\text { Patients with ane- } \\
\text { mia }(n=4434)\end{array}$ & $p$ value \\
\hline \multicolumn{5}{|l|}{ Demographics } \\
\hline Age & $67.5 \pm 15.1$ & $64.8 \pm 15.0$ & $70.6 \pm 14.7$ & $<0.001$ \\
\hline Female gender & $3604(37.4 \%)$ & $1448(27.8 \%)$ & $2156(48.6 \%)$ & $<0.001$ \\
\hline White race & $8911(92.4 \%)$ & $4860(93.3 \%)$ & $4051(91.4 \%)$ & $<0.001$ \\
\hline BMI $\left(\mathrm{kg} / \mathrm{m}^{2}\right)$ & $29.6 \pm 7.0$ & $29.8 \pm 6.8$ & $29.3 \pm 7.3$ & $<0.001$ \\
\hline CICU length of stay & $2.6 \pm 4.6$ & $2.4 \pm 4.1$ & $2.8 \pm 5.2$ & $<0.001$ \\
\hline Hospital length of stay & $8.1 \pm 13.3$ & $6.4 \pm 9.0$ & $10.0 \pm 16.9$ & $<0.001$ \\
\hline CICU mortality & $519(5.4 \%)$ & $229(4.4 \%)$ & $290(6.5 \%)$ & $<0.001$ \\
\hline Hospital mortality & $845(8.8 \%)$ & $342(6.6 \%)$ & $503(11.3 \%)$ & $<0.001$ \\
\hline \multicolumn{5}{|l|}{ Severity of illness } \\
\hline APACHE-III score & $61.7 \pm 25.2$ & $56.3 \pm 24.8$ & $68.0 \pm 24.2$ & $<0.001$ \\
\hline APACHE-IV predicted mortality & $0.172 \pm 0.202$ & $0.142 \pm 0.193$ & $0.207 \pm 0.206$ & $<0.001$ \\
\hline Day 1 SOFA score & $3.6 \pm 3.2$ & $3.1 \pm 3.1$ & $4.3 \pm 3.3$ & $<0.001$ \\
\hline Maximum week 1 SOFA score & $4.1 \pm 3.4$ & $3.5 \pm 3.2$ & $4.8 \pm 3.5$ & $<0.001$ \\
\hline Mean week 1 SOFA score & $3.1 \pm 2.7$ & $2.6 \pm 2.5$ & $3.6 \pm 2.8$ & $<0.001$ \\
\hline \multicolumn{5}{|l|}{ Comorbidities } \\
\hline Charlson comorbidity index & $2.4 \pm 2.6$ & $1.7 \pm 2.2$ & $3.2 \pm 2.9$ & $<0.001$ \\
\hline Prior myocardial infarction & $1919(19.9 \%)$ & $923(17.8 \%)$ & $996(22.5 \%)$ & $<0.001$ \\
\hline Prior heart failure & $1901(19.5 \%)$ & $709(13.6 \%)$ & $1192(26.9 \%)$ & $<0.001$ \\
\hline Prior stroke & $1179(12.3 \%)$ & $508(9.8 \%)$ & $671(15.2 \%)$ & $<0.001$ \\
\hline Prior chronic kidney disease & $1971(20.5 \%)$ & $603(11.6 \%)$ & $1368(30.9 \%)$ & $<0.001$ \\
\hline Prior diabetes mellitus & $2752(28.6 \%)$ & $1163(22.4 \%)$ & $1589(35.9 \%)$ & $<0.001$ \\
\hline Prior cancer & $2053(21.3 \%)$ & $868(16.7 \%)$ & $1185(26.8 \%)$ & $<0.001$ \\
\hline Prior lung disease & $1880(19.5 \%)$ & $835(16.1 \%)$ & $1045(23.6 \%)$ & $<0.001$ \\
\hline Prior liver disease & $190(2.0 \%)$ & $64(1.2 \%)$ & $126(2.8 \%)$ & $<0.001$ \\
\hline Prior dialysis & $562(5.8 \%)$ & $144(2.8 \%)$ & $418(9.4 \%)$ & $<0.001$ \\
\hline \multicolumn{5}{|l|}{ Discharge ICD-9 diagnoses } \\
\hline Shock & $1032(10.7 \%)$ & $454(8.7 \%)$ & $578(13.0 \%)$ & $<0.001$ \\
\hline Cardiogenic shock & $809(8.4 \%)$ & $390(7.5 \%)$ & $419(9.5 \%)$ & $<0.001$ \\
\hline Cardiac arrest & $769(8.0 \%)$ & $427(8.2 \%)$ & $342(7.7 \%)$ & 0.38 \\
\hline Acute coronary syndrome & $4143(43.0 \%)$ & $2574(49.5 \%)$ & $1569(35.4 \%)$ & $<0.001$ \\
\hline Heart failure & $3778(39.2 \%)$ & $1617(31.1 \%)$ & $2161(48.8 \%)$ & $<0.001$ \\
\hline Atrial fibrillation & $3076(31.9 \%)$ & $1380(26.5 \%)$ & $1696(38.3 \%)$ & $<0.001$ \\
\hline Any organ failure & $3471(36.0 \%)$ & $1388(26.7 \%)$ & $2083(47.0 \%)$ & $<0.001$ \\
\hline Multi-organ failure & $1549(16.1 \%)$ & $607(11.7 \%)$ & $942(21.3 \%)$ & $<0.001$ \\
\hline Respiratory failure & $1845(19.1 \%)$ & $812(15.6 \%)$ & $1033(23.3 \%)$ & $<0.001$ \\
\hline Acute kidney injury & $2013(20.9 \%)$ & $666(12.8 \%)$ & $1347(30.4 \%)$ & $<0.001$ \\
\hline Sepsis & $649(6.7 \%)$ & $186(3.6 \%)$ & $463(10.4 \%)$ & $<0.001$ \\
\hline Gastrointestinal bleeding & $398(4.1 \%)$ & $104(2.0 \%)$ & $294(6.6 \%)$ & $<0.001$ \\
\hline Acute blood loss anemia & $452(4.7 \%)$ & $135(2.6 \%)$ & $317(7.2 \%)$ & $<0.001$ \\
\hline Procedural bleeding & $725(7.5 \%)$ & $341(6.6 \%)$ & $384(8.7 \%)$ & $<0.001$ \\
\hline \multicolumn{5}{|l|}{ Therapies and procedures } \\
\hline Invasive ventilator & $1581(16.4 \%)$ & $774(14.9 \%)$ & $807(18.2 \%)$ & $<0.001$ \\
\hline Noninvasive ventilator & $1474(15.3 \%)$ & $635(12.2 \%)$ & $839(18.9 \%)$ & $<0.001$ \\
\hline Vasoactive drugs & $2407(25.0 \%)$ & $1136(21.8 \%)$ & $1271(28.7 \%)$ & $<0.001$ \\
\hline$>1$ vasoactive drug & $1148(11.9 \%)$ & $522(10.0 \%)$ & $626(14.1 \%)$ & $<0.001$ \\
\hline Dialysis & $479(5.0 \%)$ & $160(3.1 \%)$ & $319(7.2 \%)$ & $<0.001$ \\
\hline Coronary angiogram & $5092(52.8 \%)$ & $3182(61.1 \%)$ & $1910(43.1 \%)$ & $<0.001$ \\
\hline PCI & $3345(34.7 \%)$ & $2124(40.8 \%)$ & $1221(27.5 \%)$ & $<0.001$ \\
\hline Intra-aortic balloon pump & $854(8.9 \%)$ & $483(9.3 \%)$ & $371(8.4 \%)$ & 0.12 \\
\hline
\end{tabular}


Table 1 (continued)

\begin{tabular}{llllc}
\hline & $\begin{array}{l}\text { Overall popula- } \\
\text { tion }(n=9644)\end{array}$ & $\begin{array}{l}\text { Patients without } \\
\text { anemia }(n=5210)\end{array}$ & $\begin{array}{l}\text { Patients with ane- } \\
\text { mia }(n=4434)\end{array}$ & $p$ value \\
\hline $\begin{array}{l}\text { Pulmonary artery catheter } \\
\text { Red blood cell transfusion }\end{array}$ & $\begin{array}{l}721(7.5 \%) \\
1170(12.1 \%)\end{array}$ & $\begin{array}{l}339(6.5 \%) \\
198(3.8 \%)\end{array}$ & $\begin{array}{l}382(8.6 \%) \\
972(21.9 \%)\end{array}$ & $<0.001$ \\
Admission laboratory values & & & & $<0.001$ \\
Admission hemoglobin (g/dL) & $12.1 \pm 2.1$ & $13.7 \pm 1.3$ & $10.3 \pm 1.2$ & $<0.001$ \\
Admission MCV (fL) & $90.7 \pm 6.0$ & $90.8 \pm 5.2$ & $90.6 \pm 6.9$ & 0.06 \\
Microcytosis (MCV <80 fL) & $354(3.7 \%)$ & $94(1.8 \%)$ & $258(6.0 \%)$ & $<0.001$ \\
Macrocytosis (MCV > 100 fL) & $526(5.6 \%)$ & $207(4.1 \%)$ & $391(7.4 \%)$ & $<0.001$ \\
Admission RDW (\%) & $14.8 \pm 2.2$ & $14.0 \pm 1.6$ & $15.6 \pm 2.5$ & $<0.001$ \\
\hline
\end{tabular}

Data represented as mean \pm standard deviation for continuous variables and number (percent) for categorical variables. $p$ value is for the comparison of patients with and without anemia, using the Student's $t$ test for continuous variables and Chi-squared test for categorical variables

$A P A C H E$ acute physiology and chronic health evaluation, BMI body mass index, CICU cardiac intensive care unit, $I C D$ International Classification of Diseases, $M C V$ mean corpuscular volume, $P C I$ percutaneous coronary intervention, $R D W$ red cell distribution width, $S O F A$ sequential organ failure assessment

\section{Unadjusted hospital mortality}

A total of 519 (5.4\%) patients died in the CICU, and 845 (8.8\%) patients died in the hospital. Unadjusted CICU mortality was higher in patients with anemia (6.5\% vs. $4.4 \%$, unadjusted OR $1.52,95 \%$ CI $1.28-1.82, p<0.001)$, as was unadjusted hospital mortality $(11.3 \%$ vs. $6.6 \%$, unadjusted OR 1.82, 95\% CI 1.58-2.10, $p<0.001)$. Similar results were observed when using the WHO gender-specific definition of anemia: both CICU mortality (6.4\% vs. $4.1 \%$, unadjusted OR $1.58,95 \%$ CI $1.31-1.90, p<0.001)$ and hospital mortality ( $10.8 \%$ vs. $6.2 \%$, unadjusted OR $1.84,95 \%$ CI $1.58-2.14, p<0.001)$ were higher in patients with anemia. There was a stepwise increase in hospital mortality with decreasing admission $\mathrm{Hb}: \geq 12 \mathrm{~g} / \mathrm{dL}, 6.6 \%$; $10.0-11.9 \mathrm{~g} / \mathrm{dL}, 10.1 \% ; 8.0-9.9 \mathrm{~g} / \mathrm{dL}, 13.0 \% ;<8 \mathrm{~g} / \mathrm{dL}$ $16.4 \%(p<0.001)$. Admission $\mathrm{Hb}$ was inversely associated with hospital mortality (unadjusted OR 0.87 per $1 \mathrm{~g} /$ $\mathrm{dL}, 95 \%$ CI $0.84-0.90, p<0.001)$, with an optimal cutoff of $11.5 \mathrm{~g} / \mathrm{dL}$ for predicting hospital mortality. The unadjusted OR value for admission $\mathrm{Hb}$ was 0.84 per $1 \mathrm{~g} / \mathrm{dL}$ (95\% CI 0.78-0.90) among patients with anemia and 1.08 per $1 \mathrm{~g} / \mathrm{dL}$ (95\% CI 1.00-1.17) in patients without anemia, reflecting a $\mathrm{U}$-shaped relationship between admission $\mathrm{Hb}$ and mortality (Fig. 1). Admission MCV was positively associated with hospital mortality (unadjusted OR 1.04
Fig. 1 CICU and hospital mortality as a function of admission hemoglobin. $p<0.001$ for both CICU and hospital mortality across groups using Chi-squared test. $C I C U$ cardiac intensive care unit

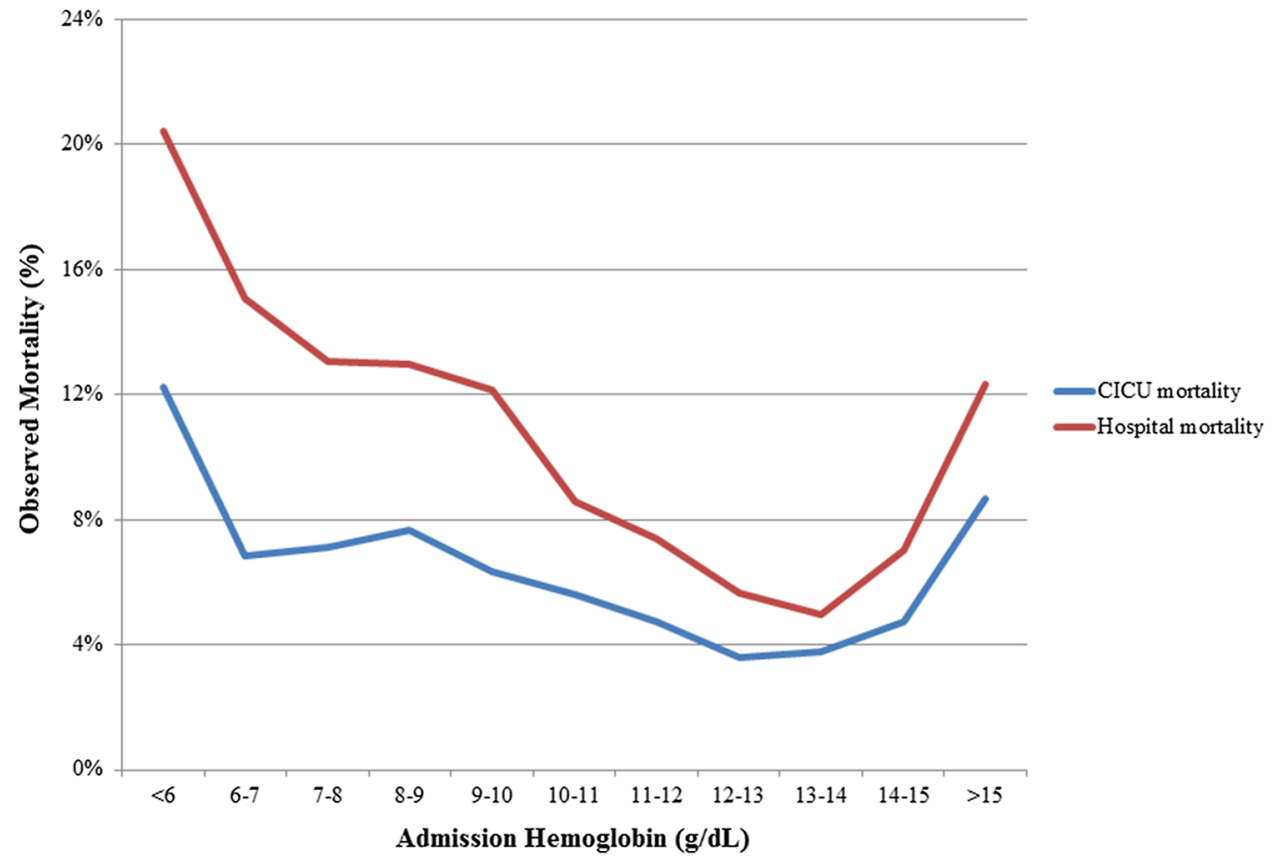


per $1 \mathrm{fL}, 95 \%$ CI $1.02-1.05 p<0.001$ ), with an optimal cutoff of $94.6 \mathrm{fL}$ for predicting hospital mortality. This relationship was similar among patients with anemia (OR 1.03 per $1 \mathrm{fL}, 95 \%$ CI $1.02-1.05)$ and patients without anemia (OR 1.04 per $1 \mathrm{fL}, 95 \%$ CI 1.01-1.06). A J-shaped relationship was observed between admission MCV and mortality (Fig. 2a); the MCV was associated with hospital mortality in patients with and without anemia, with mortality being highest in patients with macrocytic anemia (Fig. 2b). Admission RDW was positively associated with hospital mortality (unadjusted OR 1.20 per $1 \%, 95 \%$ CI $1.17-1.23, p<0.001$ ), with an optimal cutoff of $14.3 \%$ for predicting hospital mortality. This association that appeared to be stronger in patients without anemia (unadjusted OR 1.34 per $1 \%, 95 \%$ CI 1.28-1.41) compared to patients with anemia (unadjusted OR 1.11 per $1 \%, 95 \%$ CI 1.08-1.15). A progressive increase in mortality was observed with increasing decile of RDW, both in patients with and without anemia (Fig. 3).
Fig. 2 a CICU and hospital mortality as a function of admission MCV. $p<0.001$ for both CICU and hospital mortality across groups using Chi-squared test. $C I C U$ cardiac intensive care unit, $M C V$ mean corpuscular volume. b CICU and hospital mortality in patients with microcytosis $(\mathrm{MCV}<80 \mathrm{fL})$, macrocytosis $(\mathrm{MCV}>100 \mathrm{fL})$ and normocytosis (MCV 80-100 fL), with and without anemia. $p<0.001$ for both CICU and hospital mortality between groups using Chi-squared test. $C I C U$ cardiac intensive care unit, $M C V$ mean corpuscular volume
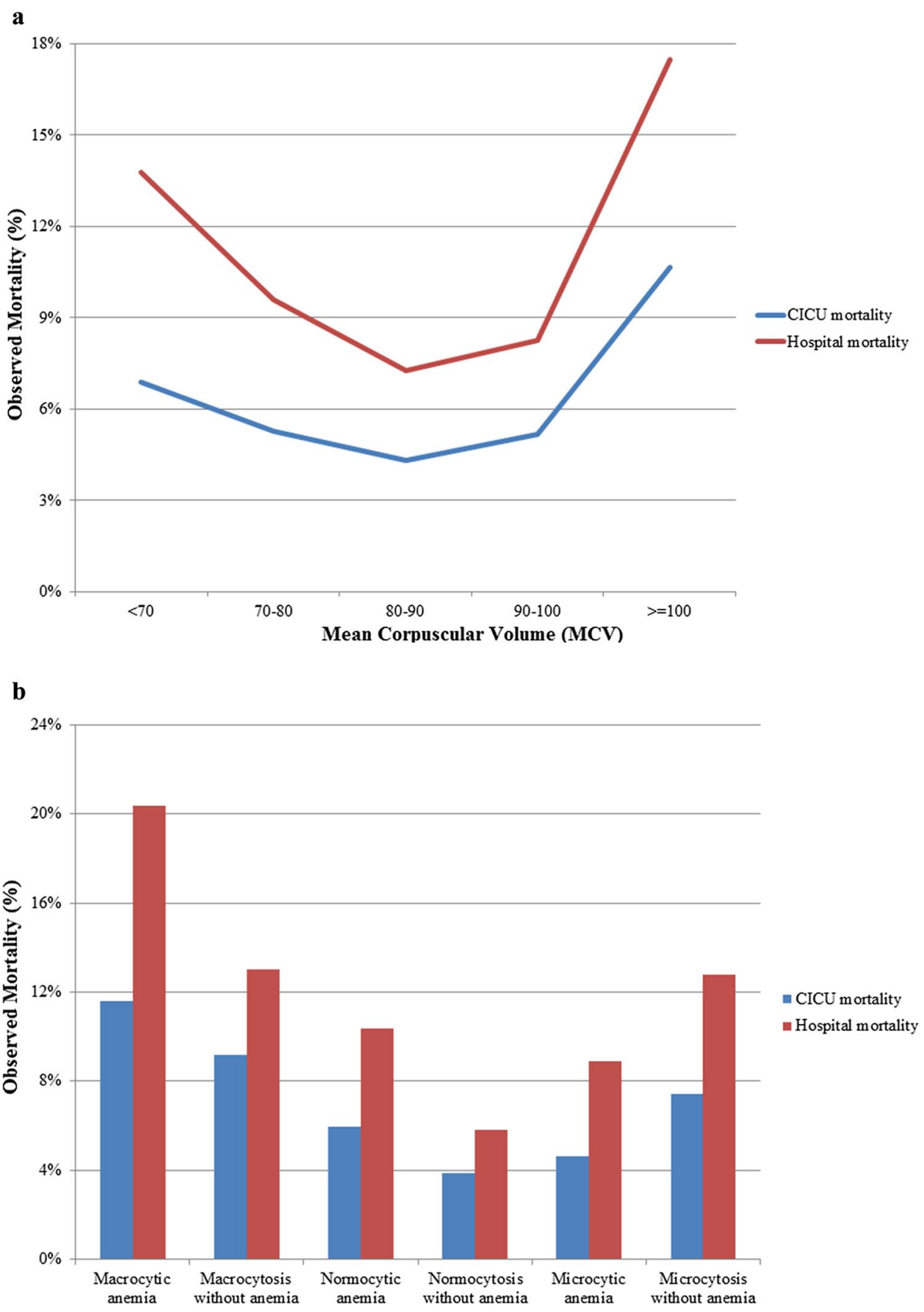
Fig. 3 Hospital mortality as a function of admission RDW decile in the overall population, patients with anemia, and patients without anemia. $p<0.001$ for hospital mortality trends across RDW declines in each group. $R D W$ red cell distribution width

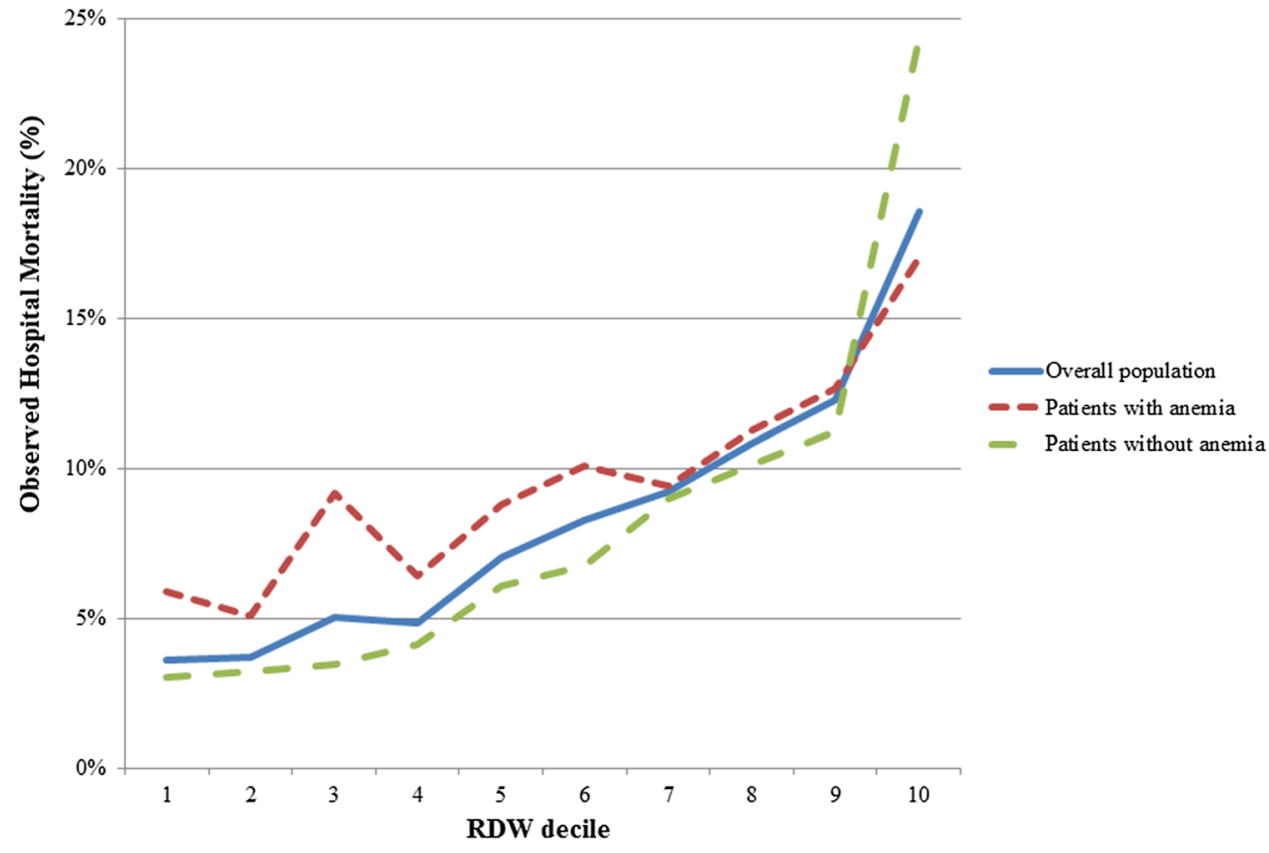

\section{Adjusted hospital mortality}

On multivariable regression adjusting for demographics, comorbidities, vital signs, laboratory data, discharge diagnoses and APACHE-III score (Table 2), neither admission $\mathrm{Hb}$ level nor anemia (both $p>0.99$ ) was associated with hospital mortality. Likewise, admission MCV (adjusted OR 1.01 per $1 \mathrm{fL}, 95 \%$ CI 1.00-1.03, $p=0.11$ ) was not significantly associated with hospital mortality. By contrast, admission RDW remained strongly associated with hospital mortality after multivariable adjustment (adjusted OR 1.15 per $1 \%$, 95\% CI 1.10-1.21, $p<0.001)$. There was a positive statistical interaction between admission $\mathrm{Hb}$ and RDW $(p=0.005)$.

\section{Post-discharge survival}

Among 8799 (91.2\%) patients surviving to hospital discharge, $3537(40.2 \%)$ died during a mean follow-up of 3.4 years; 1229 (14.0\%) hospital survivors had post-discharge follow-up less than 1 year, including loss to followup. Post-discharge survival decreased in a stepwise fashion as a function of decreasing quartile of admission $\mathrm{Hb}$ (Fig. 4a, $p<0.001$ by log-rank). Post-discharge survival differed based on the presence or absence of anemia and the admission MCV (Fig. $4 \mathrm{~b}, p<0.001$ by log-rank); survival was highest among patients with normocytosis or microcytosis without anemia and lowest among patients with macrocytic anemia. Post-discharge survival decreased in a stepwise fashion as a function of increasing quartile of admission $\mathrm{Hb}$ (Fig. $4 \mathrm{c}, p<0.001$ by log-rank). On Cox proportionalhazards analysis adjusting for predictors of hospital mortality (Table 2), admission Hb (adjusted HR 0.92 per $1 \mathrm{~g} / \mathrm{dL}$,
95\% CI 0.90-0.94), MCV (adjusted HR 1.02 per $1 \mathrm{fL}, 95 \%$ CI 1.01-1.02), and RDW (adjusted HR per 1\% 1.13, 95\% CI $1.11-1.15)$ were all significantly $(p<0.001)$ associated with post-discharge mortality among hospital survivors.

\section{Discussion}

To our knowledge, this is the largest study to examine the associations between hematologic indices with hospital and post-discharge mortality in unselected CICU patients. Anemia (admission $\mathrm{Hb}<12 \mathrm{~g} / \mathrm{dL}$ ) was present in almost $50 \%$ of patients in this CICU population and predicted higher unadjusted hospital and post-discharge mortality. Admission $\mathrm{Hb}$ demonstrated a reverse J-shaped relationship with hospital mortality, but this relationship did not persist after multivariable adjustment. Admission MCV showed a J-shaped association with hospital mortality, and patients with macrocytic anemia were at higher risk of hospital and post-discharge death. An elevated admission RDW was associated with higher hospital and post-discharge mortality, and RDW remained a significant predictor of hospital mortality after multivariable adjustment. The association between RDW and mortality was influenced by the presence or absence of anemia, as evidenced by the significant statistical interaction term on multivariable regression. The relationship between RDW and mortality appeared stronger in patients without anemia, suggesting that elevated RDW was a relevant prognostic factor and not merely a marker of anemia. Admission $\mathrm{Hb}, \mathrm{MCV}$, and RDW were all significantly associated with adjusted post-discharge mortality among hospital survivors. Patients with HF had lower $\mathrm{Hb}$ and higher RDW, as opposed 
Table 2 Predictors of hospital mortality using multivariable regression using an adaptive elastic net penalty algorithm and predictors of postdischarge mortality using Cox proportional-hazards analysis

\begin{tabular}{|c|c|c|c|c|c|c|}
\hline \multirow[t]{2}{*}{ Variable } & \multicolumn{3}{|c|}{ Inpatient mortality (regression) } & \multicolumn{3}{|c|}{ Post-discharge mortality (Cox) } \\
\hline & Adjusted OR & $95 \% \mathrm{CI}$ & $p$ value & Adjusted HR & $95 \%$ CI & $p$ value \\
\hline \multicolumn{7}{|l|}{ Comorbidities and severity of illness } \\
\hline Age (per 1 year) & 1.03 & $1.02-1.04$ & $<0.001$ & 1.03 & $1.02-1.03$ & $<0.001$ \\
\hline Inpatient days prior to $\mathrm{CICU}$ & 1.01 & $0.98-1.03$ & 0.63 & 1.00 & $0.98-1.01$ & 0.63 \\
\hline Charlson Comorbidity Index & 1.02 & $0.98-1.07$ & 0.31 & 1.12 & $1.10-1.14$ & $<0.001$ \\
\hline Prior stroke & 1.04 & $0.74-1.44$ & 0.83 & 1.02 & $0.91-1.14$ & 0.74 \\
\hline APACHE-III score (per 1 unit) & 1.02 & $1.02-1.03$ & $<0.001$ & 1.01 & $1.00-1.01$ & $<0.001$ \\
\hline \multicolumn{7}{|l|}{ Admission vital signs } \\
\hline Systolic BP (per $1 \mathrm{mmHg}$ ) & 1.00 & $0.99-1.00$ & 0.13 & 1.00 & $1.00-1.00$ & 0.02 \\
\hline Heart rate (per $1 \mathrm{BPM})$ & 1.01 & $1.00-1.01$ & 0.02 & 1.00 & $1.00-1.00$ & 0.01 \\
\hline Respiratory rate (per $1 \mathrm{BPM}$ ) & 1.01 & $0.99-1.03$ & 0.47 & 1.01 & $1.00-1.02$ & 0.02 \\
\hline \multicolumn{7}{|l|}{ Admission laboratory values } \\
\hline Hemoglobin $(\text { per } 1 \mathrm{~g} / \mathrm{dL})^{*}$ & -- & -- & -- & 0.92 & $0.90-0.94$ & $<0.001$ \\
\hline $\mathrm{MCV}$ (per $1 \mathrm{fL}$ ) & 1.01 & $1.00-1.03$ & 0.11 & 1.02 & $1.01-1.02$ & $<0.001$ \\
\hline RDW (per 1\%) & 1.15 & $1.10-1.21$ & $<0.001$ & 1.13 & $1.11-1.15$ & $<0.001$ \\
\hline Anion gap (per $1 \mathrm{mEq} / \mathrm{L}$ ) & 1.04 & $1.01-1.07$ & 0.008 & 0.99 & $0.98-1.00$ & 0.21 \\
\hline Chloride (per $1 \mathrm{mEq} / \mathrm{L}$ ) & 0.96 & $0.94-0.98$ & $<0.001$ & 0.97 & $0.96-0.98$ & $<0.001$ \\
\hline Neutrophil count (per $1000 / \mathrm{mm}^{3}$ ) & 1.03 & $1.01-1.07$ & 0.002 & 1.00 & $0.99-1.01$ & 0.89 \\
\hline BUN (per 1 mg/dL) & 1.01 & $1.00-1.01$ & 0.06 & 1.00 & $1.00-1.01$ & $<0.001$ \\
\hline \multicolumn{7}{|l|}{ Procedures and therapies } \\
\hline \# vasoactive drugs (per 1 drug) & 1.45 & $1.30-1.62$ & $<0.001$ & 1.04 & $0.98-1.11$ & 0.17 \\
\hline Dialysis in CICU & 2.00 & $1.35-2.96$ & $<0.001$ & 1.58 & $1.33-1.88$ & $<0.001$ \\
\hline Pulmonary artery catheter & 0.75 & $0.51-1.09$ & 0.14 & 0.86 & $0.73-1.02$ & 0.08 \\
\hline Coronary angiogram & 0.93 & $0.70-1.23$ & 0.59 & 0.91 & $0.82-1.01$ & 0.08 \\
\hline PCI & 0.74 & $0.54-1.01$ & 0.05 & 0.80 & $0.71-0.89$ & $<0.001$ \\
\hline \multicolumn{7}{|l|}{ Discharge ICD9 diagnoses } \\
\hline Shock & 1.32 & $0.94-1.85$ & 0.11 & 0.96 & $0.82-1.13$ & 0.66 \\
\hline Hypertension & 0.55 & $0.43-0.70$ & $<0.001$ & 0.84 & $0.77-0.91$ & $<0.001$ \\
\hline Cardiomyopathy & 0.76 & $0.56-1.04$ & 0.08 & 1.02 & $0.90-1.16$ & 0.73 \\
\hline Heart failure & 0.82 & $0.63-1.06$ & 0.14 & 1.26 & $1.14-1.39$ & $<0.001$ \\
\hline Atrial fibrillation & 0.79 & $0.63-1.01$ & 0.06 & 1.10 & $1.01-1.20$ & 0.03 \\
\hline Cardiac arrest & 3.56 & $2.59-4.89$ & $<0.001$ & 1.00 & $0.82-1.20$ & 0.96 \\
\hline Acute coronary syndrome & 1.08 & $0.79-1.48$ & 0.63 & 1.08 & $0.97-1.21$ & 0.17 \\
\hline Coronary artery disease & 0.69 & $0.53-0.90$ & 0.007 & 0.94 & $0.85-1.04$ & 0.22 \\
\hline Acute kidney injury & 1.06 & $0.81-1.37$ & 0.69 & 1.02 & $0.91-1.12$ & 0.75 \\
\hline Respiratory failure & 2.21 & $1.68-2.90$ & $<0.001$ & 1.18 & $1.06-1.32$ & 0.003 \\
\hline Procedural bleeding & 1.47 & $0.97-2.23$ & 0.07 & 0.82 & $0.69-0.97$ & 0.02 \\
\hline
\end{tabular}

Adjusted odds ratio (OR) and $95 \%$ confidence interval (CI) values are shown

$A P A C H E$ acute physiology and chronic health evaluation, BUN blood urea nitrogen, $C I C U$ cardiac intensive care unit, $M C V$ mean corpuscular volume, $P C I$ percutaneous coronary intervention, $R D W$ red cell distribution width

*Admission $\mathrm{Hb}$ was not selected for inclusion in the final regression model for hospital mortality

to patients with ACS, implying an association between myocardial dysfunction and these hematologic abnormalities. These data emphasize the importance of easily-available hematologic laboratory data for risk stratification of CICU patients and highlight the interactions between hematologic abnormalities and acute cardiovascular disease outcomes.
Anemia has been associated with adverse outcomes across a broad spectrum of patients with acute cardiovascular disease and critical illness [1-5, 15]. We observed the same curvilinear relationship between admission $\mathrm{Hb}$ and unadjusted hospital mortality in our CICU population, as was previously reported in ACS patients [4]. However, after 

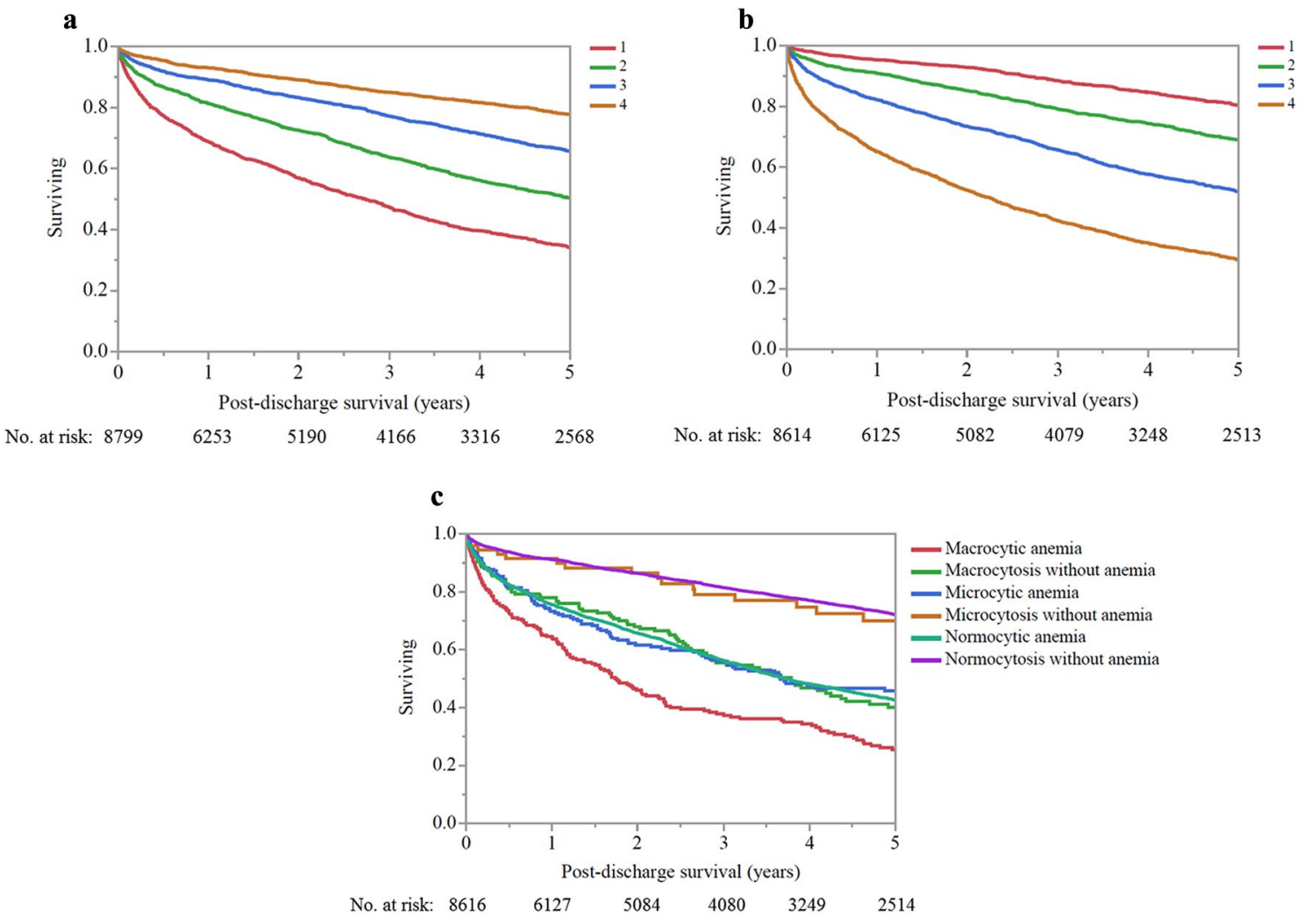

Fig. 4 a Kaplan-Meier curves demonstrating post-hospital survival among hospital survivors, grouped by admission hemoglobin quartile, based on a median of 12.2 (IQR 10.6-13.7) g/dL. $p<0.001$ between groups by log-rank. b Kaplan-Meier curves demonstrating post-hospital survival among hospital survivors, grouped by RDW quartile based on a mean RDW of $14.1 \%$ (IQR 13.3-15.6). $p<0.001$

multivariable adjustment we did not observe an association between admission $\mathrm{Hb}$ and hospital mortality in this large CICU population, potentially suggesting that anemia may be a marker of sicker CICU patients rather than a directly pathological entity in many cases [15]. Our findings imply that acutely raising the $\mathrm{Hb}$ level, for instance by transfusion, may not necessarily reduce mortality in CICU patients, consistent with prior studies that have failed to demonstrate improved outcomes using higher $\mathrm{Hb}$ thresholds for transfusion among patients with acute cardiovascular disease [28]. This highlights the importance of ongoing studies such as the Myocardial Ischemia and Transfusion trial (MINT, NCT02981407) to define the role of transfusion in acutely-ill cardiac patients with anemia.

Unlike our results for hospital mortality, a lower admission $\mathrm{Hb}$ was associated with higher post-discharge mortality among hospital survivors. A prior study by Uscinska, et al. found that lower $\mathrm{Hb}$ levels and decreased markers of iron stores were associated with lower long-term survival in their cohort of 392 CICU patients [7]. Anemia and iron deficiency have been identified as relevant prognostic variables between groups by log-rank. RDW, red cell distribution width. c Kaplan-Meier curves demonstrating post-hospital survival among hospital survivors, grouped by admission MCV and the presence of anemia (admission hemoglobin $<12 \mathrm{~g} / \mathrm{dL}$ ). $p<0.001$ between groups by log-rank. $M C V$ mean corpuscular volume

and important targets for therapy in patients with HF [29, 30]. Chronic iron deficiency typically produces microcytosis with an elevated RDW, although macrocytosis can be seen during acute blood loss due to regenerative reticulocytosis [30]. In our CICU population, macrocytosis appeared to be more strongly associated with adverse outcomes than microcytosis, raising important questions about the underlying pathophysiology that drives the associations between anemia, MCV and outcomes.

The association between MCV and mortality has not been previously explored in unselected CICU patients, and our results suggest that an elevated MCV is more significantly associated with post-discharge than hospital mortality. Huang et al. demonstrated an association between higher MCV and higher RDW with increased unadjusted hospital mortality among acute MI patients in the ICU; as in our study, $\mathrm{Hb}$ levels were associated with unadjusted mortality but not adjusted mortality [12]. The mean corpuscular $\mathrm{Hb}$ concentration was inversely related to adjusted hospital mortality in their population, and RDW was associated with adjusted 1-year mortality. Similarly, Ueda et al. 
demonstrated that an elevated MCV is associated with mortality among patients with acute HF [6]. Prior studies have shown associations between elevated MCV with mortality and ICU readmission in other ICU populations [14, 16]. Bazick et al. previously demonstrated a higher MCV to be associated with increased hospital mortality in a cohort of 51,143 mixed ICU patients (including 15\% with MI and $22 \%$ with HF), after performing multivariable adjustment [16]. Numerous potential pathophysiologic mechanisms may explain the association between elevated MCV and adverse outcomes, including altered hematopoiesis due to intrinsic bone marrow abnormalities, organ dysfunction, drugs, toxins, and nutritional deficiency which may influence macrocyte morphology and produce concomitant cytopenias [23]. An elevated MCV may represent myelodysplastic syndrome or clonal hematopoiesis of indeterminate potential (CHIP), the latter of which has been associated with an increased risk of cardiovascular events and highlighted as a potent emerging cardiovascular risk factor [31, 32].

RDW was one of the most significant predictors of the adjusted hospital and post-discharge mortality in this CICU cohort and appeared to be more strongly associated with mortality among patients without anemia, even though patients with anemia had higher RDW. These findings in a mixed CICU population may be expected given the consistent association between an elevated RDW and increased mortality in patients with acute cardiovascular disease and general ICU patients [9-11, 14, 16-18]. A prior study by $\mathrm{Hu}$, et al. demonstrated an association between elevated RDW with hospital mortality and AKI in 412 CICU patients, even after adjustment for illness severity and other variables, higher RDW was associated with lower long-term survival [8]. These authors found a positive correlation between RDW and the APACHE-II score, suggesting that RDW correlates with illness severity yet provides additional prognostic value. Prior studies in patients with acute myocardial infarction have consistently demonstrated that an elevated RDW is associated with higher mortality, even after adjustment for relevant risk scores [10]. Similar associations between elevated RDW and death have been reported in patients with acute HF, including patients with and without anemia [9]. In one of the largest studies examining RDW in critically ill patients, Bazick et al. reported a strong independent relationship between RDW and mortality [16]. As in our study, patients with elevated RDW were older, had greater comorbidities, more frequently had sepsis, had a worse renal function and lower Hb levels, and had a higher prevalence of organ failure.

Several potential hypotheses have been proposed to explain the association between elevated RDW and mortality, which is undoubtedly multifactorial and likely mediated by higher illness severity and worse underlying cardiac substrate $[9,10,16,17]$. The factors which may drive both altered hematopoiesis and adverse outcomes include increased inflammation or oxidative stress (as suggested by studies showing correlations between RDW and C-reactive protein) and the effects of multi-organ dysfunction (including AKI) and baseline comorbidities on bone marrow function and hematopoiesis [9, 10, 15-17]. While an elevated RDW clearly portends higher hospital and post-discharge mortality risk independent of anemia, it remains uncertain how to leverage this information to optimize treatment for CICU patients.

\section{Limitations}

This study has a number of relevant limitations that apply similarly to other retrospective cohort analyses, particularly the potential for additional unmeasured confounding variables. The CICU population at Mayo Clinic may differ from other populations in terms of baseline demographics and case mix [19]. We focused on admission laboratory values to predict mortality and were not able to determine whether changes in these variables during hospitalization influenced mortality risk. We did not have data available on pre-admission laboratory values, prior hematologic abnormalities, preceding transfusion, blood cell morphology, hemolysis, reticulocytosis, bleeding, iron studies, or vitamin levels. This prevented us from determining the causes and chronicity of anemia, elevated MCV, or elevated RDW in our patients, and from defining how these hematologic abnormalities may have led to higher mortality. Likewise, we did not have echocardiographic data available to better elucidate the relationships between HF and myocardial dysfunction with abnormal hematologic parameters. Our post-discharge mortality analysis should be considered exploratory, as the use of electronic health record review to determine patient death may underestimate post-discharge mortality by potentially failing to capture patients dying in other health systems. We did not have data available to determine cause of death, preventing us from providing specific insights about the mechanisms that could have linked abnormal hematologic indices with mortality risk.

\section{Conclusions}

Anemia is common among CICU patients and is associated with higher unadjusted short-term and long-term mortality, but this relationship seems to be mediated primarily by illness severity and comorbidities. By contrast, elevated RDW was strongly associated with higher hospital mortality after adjusting for relevant covariates, as shown in other populations of cardiac and ICU patients. Anemia, MCV, and RDW were able to risk-stratify post-discharge mortality among hospital survivors. Incorporation of RDW into 
future severity of illness scores may be useful for enhancing mortality risk stratification. Future studies are needed to understand the relationship between RDW and outcomes in CICU patients to determine whether RDW reflects specific underlying pathophysiology or simply greater illness severity and to better define the underlying pathophysiology linking abnormal hematopoiesis and outcomes in patients with acute cardiovascular disease.

Acknowledgements The authors would like to acknowledge the dedicated physicians, nurses and allied health staff comprising the multidisciplinary team that cares for our CICU patients each day.

Funding No extramural funding was involved in the conduct of this research.

\section{Compliance with ethical standards}

Conflict of interest The authors report no relevant conflicts of interest.

\section{References}

1. Lawler PR, Filion KB, Dourian T, Atallah R, Garfinkle M, Eisenberg MJ (2013) Anemia and mortality in acute coronary syndromes: a systematic review and meta-analysis. Am Heart J 165(2):143-153 e145

2. Kajimoto K, Sato N, Takano T, Investigators of the Acute Decompensated Heart Failure Syndromes r (2015) Association between anemia, clinical features and outcome in patients hospitalized for acute heart failure syndromes. Eur Heart J Acute Cardiovasc Care 4(6):568-576

3. Kwok CS, Tiong D, Pradhan A, Andreou AY, Nolan J, Bertrand OF, Curzen N, Urban P, Myint PK, Zaman AG et al (2016) Meta-analysis of the prognostic impact of anemia in patients undergoing percutaneous coronary intervention. Am J Cardiol 118(4):610-620

4. Brener SJ, Mehran R, Dangas GD, Ohman EM, Witzenbichler B, Zhang Y, Parvataneni R, Stone GW (2017) Relation of baseline hemoglobin levels and adverse events in patients with acute coronary syndromes (from the Acute Catheterization and Urgent Intervention Triage strategY and Harmonizing Outcomes with RevasculariZatiON and Stents in Acute Myocardial Infarction Trials). Am J Cardiol 119(11):1710-1716

5. Backhaus T, Fach A, Schmucker J, Fiehn E, Garstka D, Stehmeier J, Hambrecht R, Wienbergen H (2018) Management and predictors of outcome in unselected patients with cardiogenic shock complicating acute ST-segment elevation myocardial infarction: results from the Bremen STEMI Registry. Clin Res Cardiol 107(5):371-379

6. Ueda T, Kawakami R, Horii M, Sugawara Y, Matsumoto T, Okada S, Nishida T, Soeda T, Okayama S, Somekawa S et al (2013) High mean corpuscular volume is a new indicator of prognosis in acute decompensated heart failure. Circ J 77(11):2766-2771

7. Uscinska E, Sobkowicz B, Lisowska A, Sawicki R, Dabrowska M, Szmitkowski M, Musial WJ, Tycinska AM (2016) Predictors of long-term mortality in patients hospitalized in an Intensive Cardiac Care Unit. Int Heart J 57(1):67-72

8. Hu Y, Liu H, Fu S, Wan J, Li X (2017) Red blood cell distribution width is an independent predictor of AKI and mortality in patients in the Coronary Care Unit. Kidney Blood Press Res 42(6):1193-1204
9. Cheng YL, Cheng HM, Huang WM, Lu DY, Hsu PF, Guo CY, Yu WC, Chen CH, Sung SH (2016) Red cell distribution width and the risk of mortality in patients with acute heart failure with or without cardiorenal anemia syndrome. Am J Cardiol 117(3):399-403

10. Abrahan LLT, Ramos JDA, Cunanan EL, Tiongson MDA, Punzalan FER (2018) Red cell distribution width and mortality in patients with acute coronary syndrome: a meta-analysis on prognosis. Cardiol Res 9(3):144-152

11. Uemura Y, Shibata R, Takemoto K, Uchikawa T, Koyasu M, Watanabe H, Mitsuda T, Miura A, Imai R, Watarai M et al (2016) Elevation of red blood cell distribution width during hospitalization predicts mortality in patients with acute decompensated heart failure. J Cardiol 67(3):268-273

12. Huang YL, Hu ZD (2016) Lower mean corpuscular hemoglobin concentration is associated with poorer outcomes in intensive care unit admitted patients with acute myocardial infarction. Ann Transl Med 4(10):190

13. Thygesen K, Alpert JS, Jaffe AS, Chaitman BR, Bax JJ, Morrow DA, White HD (2018) Fourth universal definition of myocardial infarctio. J Am Coll Cardiol 2018:25285

14. Tonietto TA, Boniatti MM, Lisboa TC, Viana MV, Dos Santos MC, Lincho CS, Pellegrini JAS, Vidart J, Neyeloff JL, Faulhaber GAM (2018) Elevated red blood cell distribution width at ICU discharge is associated with readmission to the intensive care unit. Clin Biochem 55:15-20

15. Hayden SJ, Albert TJ, Watkins TR, Swenson ER (2012) Anemia in critical illness: insights into etiology, consequences, and management. Am J Respir Crit Care Med 185(10):1049-1057

16. Bazick HS, Chang D, Mahadevappa K, Gibbons FK, Christopher KB (2011) Red cell distribution width and all-cause mortality in critically ill patients. Crit Care Med 39(8):1913-1921

17. Luo R, Hu J, Jiang L, Zhang M (2016) Prognostic value of red blood cell distribution width in non-cardiovascular critically or acutely patients: a systematic review. PLoS ONE 11(12):e0167000

18. Fujita B, Franz M, Figulla HR, Pfeifer R, Kabisch B, Fritzenwanger M, Jung C (2015) Red cell distribution width and survival in patients hospitalized on a medical ICU. Clin Biochem 48(16-17): 1048-1052

19. Goldfarb M, van Diepen S, Liszkowski M, Jentzer JC, Pedraza I, Cercek B: Noncardiovascular Disease and Critical Care Delivery in a Contemporary Cardiac and Medical Intensive Care Unit. J Intensive Care Med 2017:885066617741873.

20. Bennett CE, Wright RS, Jentzer J, Gajic O, Murphree DH, Murphy JG, Mankad SV, Wiley BM, Bell MR, Barsness GW (2019) Severity of illness assessment with application of the APACHE IV predicted mortality and outcome trends analysis in an academic cardiac intensive care unit. J Crit Care 50:242-246

21. Jentzer JC, Bennett C, Wiley BM, Murphree DM, Keegan MT, Gajic O, Wright RS, Barsness GW (2018) Predictive value of the sequential organ failure assessment score for mortality in a contemporary cardiac intensive care unit population. J Am Heart Assoc 7(6):e008169

22. Jentzer JC, Murphree DH, Wiley B, Bennett C, Goldfarb M, Keegan MT, Murphy JG, Wright RS, Barsness GW (2018) Comparison of mortality risk prediction among patients $\% 3 \mathrm{e} /=70 \mathrm{Ver}-$ sus $\% 3 c 70$ years of age in a Cardiac Intensive Care Unit. Am J Cardiol 122(10): 1773-1778

23. Cappellini MD, Motta I (2015) Anemia in clinical practice-definition and classification: does hemoglobin change with aging? Semin Hematol 52(4):261-269

24. Chandra S, Kashyap R, Trillo-Alvarez CA, Tsapenko M, Yilmaz M, Hanson AC, Pickering BW, Gajic O, Herasevich V (2011) Mapping physicians' admission diagnoses to structured concepts towards fully automatic calculation of acute physiology and chronic health evaluation score. BMJ Open 1(2):e000216 
25. Harrison AM, Yadav H, Pickering BW, Cartin-Ceba R, Herasevich V (2013) Validation of computerized automatic calculation of the sequential organ failure assessment score. Crit Care Res Pract 2013:975672

26. Singh B, Singh A, Ahmed A, Wilson GA, Pickering BW, Herasevich V, Gajic O, Li G (2012) Derivation and validation of automated electronic search strategies to extract Charlson comorbidities from electronic medical records. Mayo Clin Proc 87(9):817-824

27. Zou H, Hastie T (2005) Regularization and variable selection via the elastic net. J Roy Stat Soc B 67:301-320

28. Hebert PC, Yetisir E, Martin C, Blajchman MA, Wells G, Marshall J, Tweeddale M, Pagliarello G, Schweitzer I, Transfusion Requirements in Critical Care Investigators for the Canadian Critical Care Trials G (2001) Is a low transfusion threshold safe in critically ill patients with cardiovascular diseases? Crit Care Med 29(2):227-234
29. Wienbergen H, Pfister O, Hochadel M, Fach A, Backhaus T, Bruder O, Remppis BA, Maeder MT, von Scheidt W, Pauschinger $M$ et al (2019) Long-term effects of iron deficiency in patients with heart failure with or without anemia: the RAID-HF follow-up study. Clin Res Cardiol 108(1):93-100

30. von Haehling S, Ebner N, Evertz R, Ponikowski P, Anker SD (2019) Iron deficiency in heart failure: an overview. JACC Heart Fail 7(1):36-46

31. Jaiswal S, Natarajan P, Silver AJ, Gibson CJ, Bick AG, Shvartz E, McConkey M, Gupta N, Gabriel S, Ardissino D et al (2017) Clonal hematopoiesis and risk of atherosclerotic cardiovascular disease. N Engl J Med 377(2):111-121

32. Libby P, Sidlow R, Lin AE, Gupta D, Jones LW, Moslehi J, Zeiher A, Jaiswal S, Schulz C, Blankstein R et al (2019) Clonal hematopoiesis: crossroads of aging, cardiovascular disease, and cancer: JACC review topic of the week. J Am Coll Cardiol 74(4):567-577 